\title{
1-D and 2-D Monte Carlo simulations for analysis of CPV module characteristics including the acceptance angle impacted by assembly errors
}

\author{
Kenji Araki , Hirokazu Nagai , Rebeca Herrero , Ignacio Antón , Gabriel Sala , Kan-Hua Lee , \\ Masafumi Yamaguchi
}

\begin{abstract}
A B S T R A C T
CPV (Concentrator photovoltaic) requires accuracy in optical alignment. Optical misalignment in the module was measured and analyzed. Such information was useful to identify assembly problems and improved module performance.

1-D and 2-D Monte Carlo simulations were developed to analyze the impact of alignment errors by assembly errors. The 1-D simulation was useful to anticipate the power output of the module, whereas $2-\mathrm{D}$ was for acceptance angle.

The 2-D model showed that the gap from Pmp-based acceptance angle (Pmp: Power at the maximum power point) from that of Isc-based (Isc: Short-circuit current) was a function of deviation of assembles misalignment in the module.

The rough rule for the acceptable assembles misalignment is that the standard deviation of the assemble misalignment will be less than $10 \%$ of acceptance angle of the optics, whereas that of power output of the single module is $20 \%$. This fact implies that the inspection of the acceptance angle is highly recommended in the CPV module production.
\end{abstract}

\section{Introduction}

HCPV (high concentrator photovoltaic) system uses optics that concentrates the sunlight onto small solar cells (Fernández et al., 2016). It is known as one of the highest-efficiency photovoltaic systems. However, it has inherent technological difficulties from optical alignment (Leutz et al., 1999).

Many CPV modules and concentrator optics tried to improve system performance by relaxing misalignment tolerance (Hall et al., 2011; Díaz et al., 2004). Although they were successful in specific product designs, systematic solution, especially in keeping or expanding acceptance angle of modules have not been done in the past. Our approach is to modify the Monte Carlo simulation that was originally developed for analysis of module performance deterioration by assembles error (Araki et al., 2016a). Although it was originally developed to anticipate module performance with given level of production control, it can be used for analysis of acceptance angle of the module with slight modification. The original Monte Carlo simulation used a 1-dimensional vector of a random number for representing lens/cell misalignment magnitude (Araki et al., 2016a). It effectively analyzed the sensitivity of the CPV module characteristics by these variances. However, the performance in the CPV system is not only affected by the variance but also the tolerance to the tracking errors. This situation is similar between the component variance and assembly errors, namely, the module characteristics can be regarded as the component variance in the module and that of the off-axis characteristics of the module can be regarded as the assembly errors in the module. Therefore, it is important to anticipate and analyze the off-axis characterization of the CPV modules with influence of component variance and assembly errors.

However, for analysis of acceptance angle, it was also necessary to consider direction of lens/cell misalignment and a group of misalignment error vector was expressed as a 2-dimensional matrix (Araki et al., 2016b). This extension was necessary because the error of the cell/lens misalignment was not always in the same direction of the tracking error. 
This study discusses the 2-D extension of the Monte Carlo simulation for the CPV module performance.

The Monte-Carlo simulation is widely used for the design of the optics for anticipating optical efficiency and performance optimization (Serrano-Aguilera et al., 2016; Ota and Nishioka, 2012). Different from this approach, our Monte-Carlo simulation does not apply to the ray-tracing and its commercial packages, but it simulates the electrical performance of CPV modules.

\section{Extension of the 1-D Monte Carlo simulation}

\subsection{1-D Monte Carlo simulation}

The detailed explanation of the 1-D Monte Carlo simulation is found in our previous work (Araki et al., 2016a). For application to the analysis on CPV modules that deals with behavior responsible from misalignment, the random number for the simulation of $\mathrm{I}-\mathrm{V}$ curve of the module were given as assemble errors and component characteristics errors. The variables of component errors were electrical characteristics errors such as short-circuit current, open-circuit voltage, diode-ideality factor, and darkcurrent and not including mechanical errors, for example, displacement. These were measured by an electrical performance test of COC (Chip-on-carrier). Errors in optical components were not considered, because their variance was much smaller than that of cells. Misalignment errors were represented by the misalignment angles as the measurement result of MOA (Araki et al., 2016a, 2016b). The displacement error could be converted to the angle error by the ratio of magnitude of displacement and its working distance.

The 1-D Monte Carlo simulation was effective to predict the $\mathrm{FF}$ and Pm of the CPV module impacted by the assembly error. One of the most important messages was the design rule that the standard deviation of misalignment should be controlled to less than $20 \%$ of the designed acceptance half angle.

Fig. 1 is the summary of the 1-D Monte Carlo simulation on the analysis of misalignment impact to the CPV module performance (Araki et al., 2016a).

\subsection{2-D axially symmetrical Monte Carlo simulation}

For analysis of acceptance angle, it was also necessary to consider direction of lens/cell misalignment and a group of misalignment error vector was expressed as a 2-dimensional matrix. This extension was necessary because the error of the cell/lens misalignment was not always in the same direction of the tracking error.

For simplicity, the case of the axially symmetrical model was discussed at first. As it is discussed later, the symmetrical model is effective to the optics with approximately symmetrical response of the output to the misalignment error. The typical example is DFK optics (Zamora Herranz et al,, 2012; Datas, 2013; Nagai et al., 2014).

The 2-D Monte Carlo simulation was developed to analyze the difference of the acceptance angle of CPV modules, especially the difference between that of Isc and Pmp (Araki et al., 2016b).

Its flow chart of the new 2-D Monte Carlo simulation is shown in Fig. 2. The comparison to the 1-D simulation is shown in Fig. 3.

The error in the 2-dimensional misalignment distribution is mainly described as the standard deviation $\sigma$. It was calculated by the measurement of MOA by Eq. (1).

$\sigma^{2}=\sigma_{x}^{2}+\sigma_{y}^{2}$

where,

$\sigma$ - standard deviation of misalignment error in the module.

$\sigma x$ - measured standard deviation along $X$-axis.

$\sigma y$ - measured standard deviation along Y-axis.

The measured distribution of the misalignment inside the real modules along both $\mathrm{X}$-axis and $\mathrm{Y}$-axis is plotted in Fig. 4.

One of the most important factors in this analysis was the characterization function of how optics behaves under off-axis conditions (i.e., the angular transmittance). Some optics may have large acceptance angle but its optical efficiency sharply drops if tracking error exceeds some certain limit. Other optics may have low acceptance tolerance but its output is still high even with
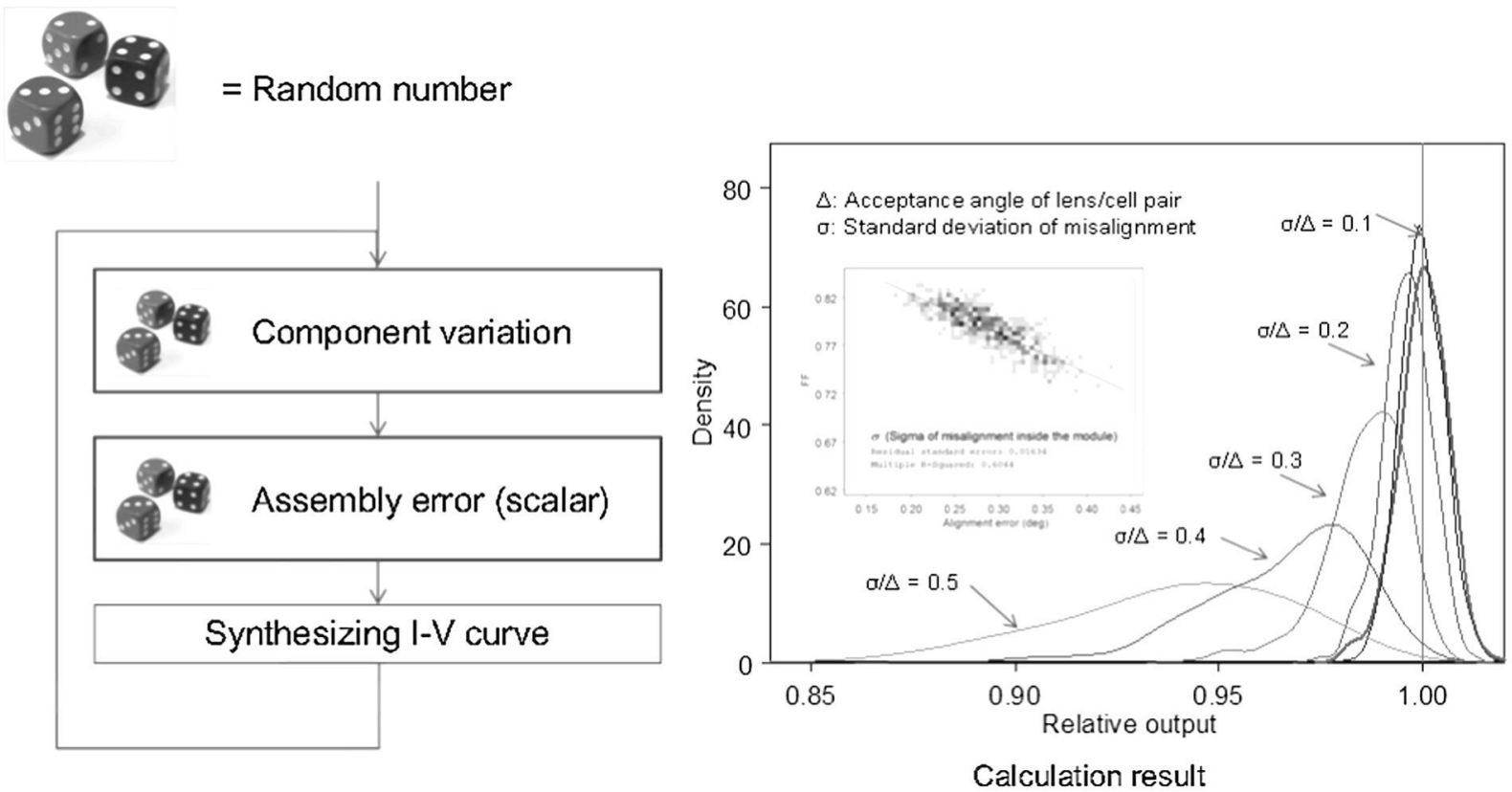

Fig. 1. 1-D Monte Carlo simulation on the misalignment analysis of CPV and its typical calculation result (Araki et al., 2016a). 


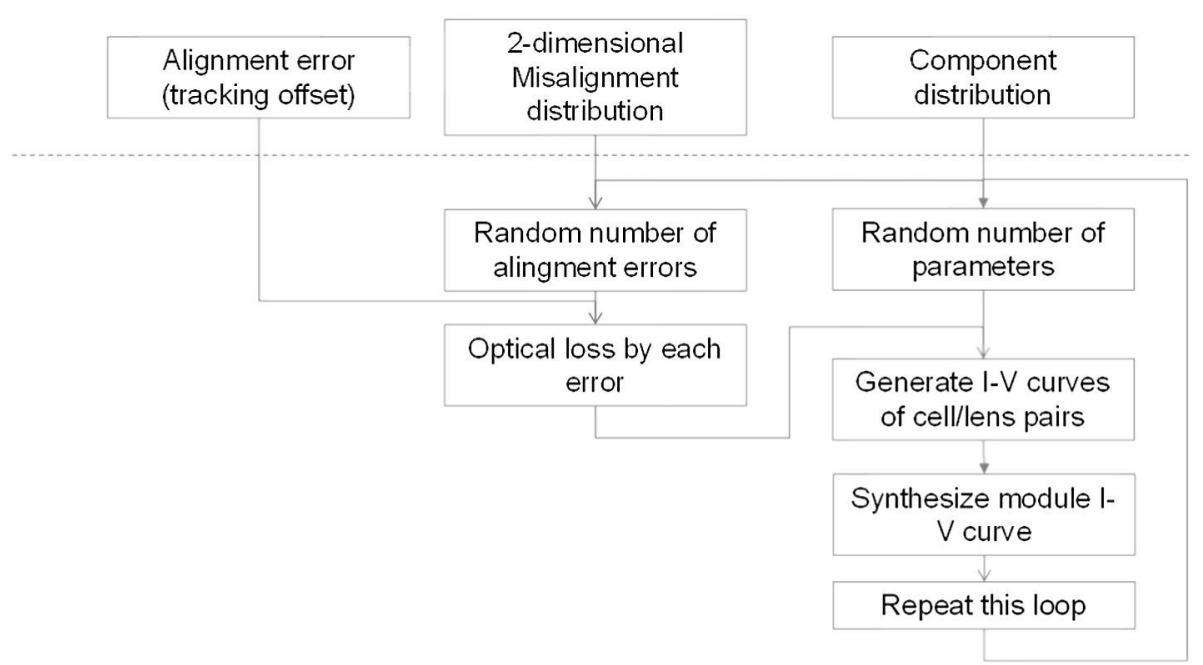

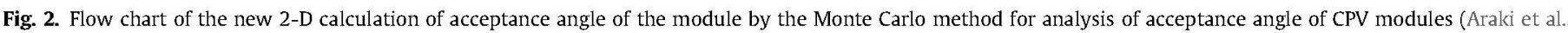
2016a).
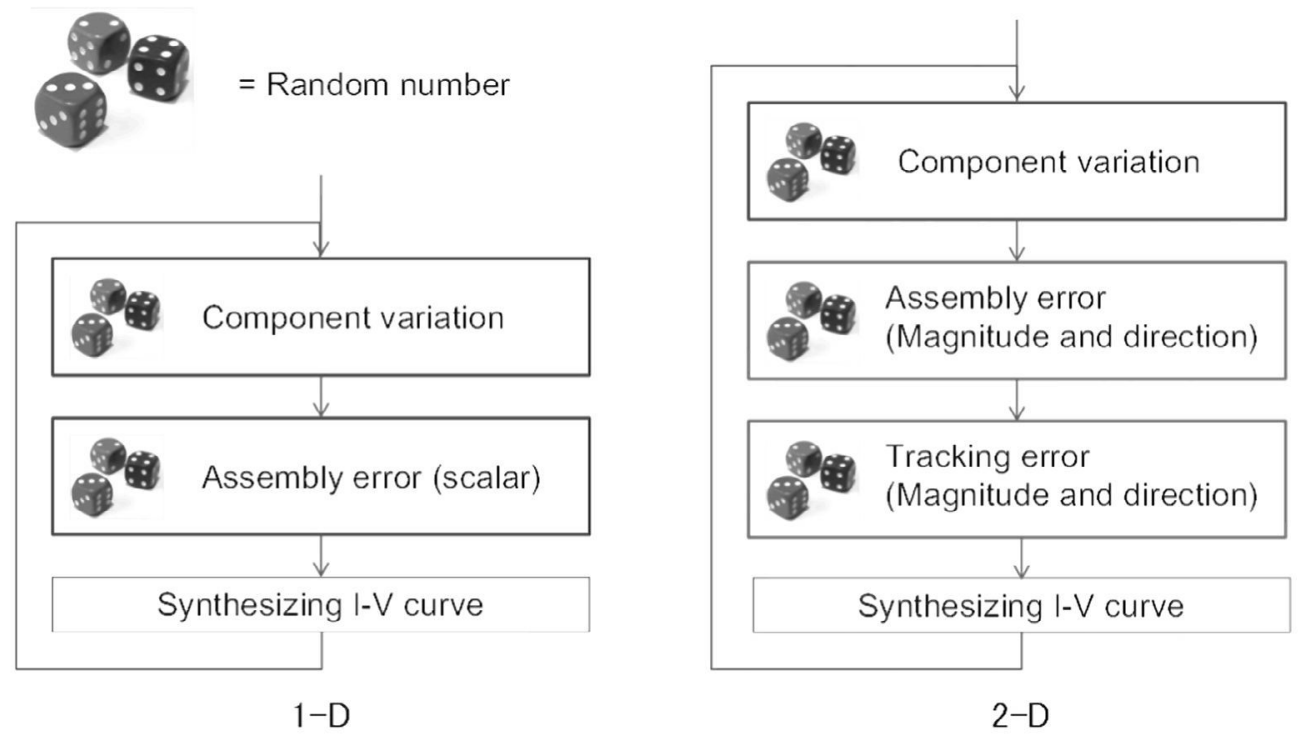

Fig. 3. Difference between 1-D and 2-D simulation.

bigger incident angle than the acceptance angle value. In order to express typical response of misaligned concentrator optics, we used a power function as Eq. (2).

$g(\delta)=\left(1-0.1\left(\frac{|\delta|}{\Delta}\right)^{m}\right)\left(0.1\left(\frac{|\delta|}{\Delta}\right)^{m}<1\right)$

where,

$g(\delta)$ - function of relative output of the optics with given $\delta$.

$\delta$ - incident angle to optics.

$\Delta$ - acceptance half angle of the optics (90\% output).

$m$ - characterization parameter of optics.

Note that incident angle of the optics $\delta$ is normalized by acceptance half angle of the optics $\Delta$.

The influence of non-uniform illumination onto concentrator cells can be included or excluded in Eq. (1). In this work, $g(\delta)$ was just fit to the trend of flux intensity. This means it was assumed that the concentration is uniform onto the cell and there will not be any additional deterioration performance by nonuniform illumination.

The parameter $\mathbf{m}$ was newly introduced in this study. This is a kind of shape factor of the angular transmittance of concentrator optics and becomes large when the optics becomes sophisticated.

It was decided by the data-fitting technique from response measurement or Ray-tracing calculation. The model equation is Eq. (3), and $\Delta$ and $m$ were numerically calculated so that the sum of square of error vector $\varepsilon$ will be minimized.

$$
\begin{aligned}
& T_{i}=\left(1-0.1\left(\frac{\left|\delta_{i}\right|}{\Delta}\right)^{m}\right)\left(0.1\left(\frac{\left|\delta_{i}\right|}{\Delta}\right)^{m}<1\right)+\varepsilon_{i} \\
& \left(\begin{array}{c}
m \\
\Delta
\end{array}\right)=\operatorname{Minimize}\left(\sum_{i} \varepsilon_{i}^{2}\right)
\end{aligned}
$$

where,

$\delta i$ - data vector of incident angle to optics.

$T i$ - data vector of relative output.

$\varepsilon i$ - error vector distributed by the normal distribution. 


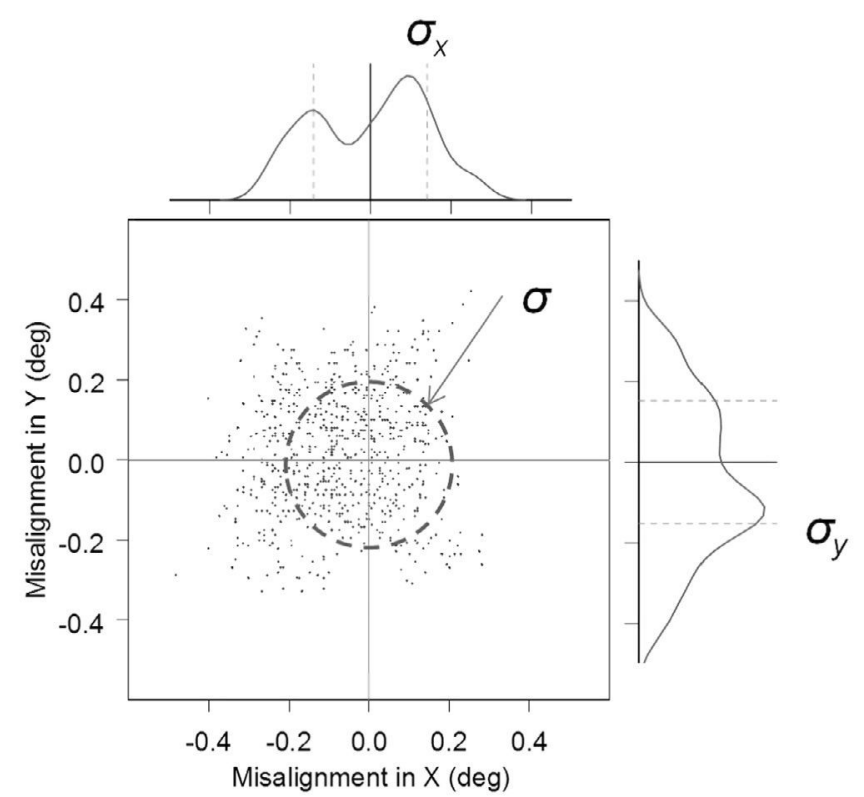

Fig. 4. Definition of the standard deviation in the 2-dimensinal alignment error distribution and the measured distribution in modules (Araki et al., 2016a).

For a simple off-focused imaging lens, $m=1$. For DFK optics, it was calculated as $m=3.445, \Delta=1$ degree in the direction parallel to cell sides, and $m=3.077, \Delta=1.05$ degree in the direction parallel to the diagonal of the cell. Note that both off-axis magnitude and directions should be necessary to considered for simulating influence of tracking error to the module performance.

It is necessary to consider the behavior of the bypass diode for ISC response related to the unit with optical errors (Herrero et al., 2010). In this paper, it was assumed that the bypass diode turns ON ideally when it was biased more than threshold voltage.

\subsection{2-D Monte Carlo simulation for highly asymmetrical acceptance angles}

The acceptance half angle of the DFK optics was almost axially symmetrical $(m=3.445, \Delta=1$ degree in the direction parallel to cell sides, and $m=3.077, \Delta=1.05$ degree in the direction parallel to the diagonal of the cell). In this case, the off-axis output in Eq. (2) can be applied to any direction of the tracking error.

However, some optics may have strong asymmetrical acceptance angles. When this happens, the parameter set $(m, \Delta)$ in Eq. (2) is a function of the directional angle. It may be represented by 2-D spline function from the datasets in 0 degree, 15 degree, ..., 45 degree, for example. In any cases, the model will be much more complicated than that of the symmetrical optics.

However, in case the simulation is to be used only for the production control, this procedure can be simplified to the calculation in the worst-case. In most of the concentrator optics with the square cell aperture, it will be the direction parallel to the side of the cell aperture.

\section{Analysis using 2-D Monte Carlo simulations}

In this chapter, the extended 2-D Monte Carlo simulation was applied to various types of CPV modules and its calculated results were compared to the measurement.

\subsection{Acceptance angle by Pmp and Isc}

The typical approach that relaxes tolerance is to expand acceptance angle of the modules. However, this "acceptance angle of the module" is confusing by double definitions, difficulty in control in module production, and difficulty in product testing (module). Taking example of measurement, it was historically done by walk-in method, so that the ISC of the module is monitored while tracker stays in the same position (Hall et al., 2011). However, it has been recognized that the acceptance angle measured by this walk-in method is too optimistic. Alternatively, Pmp or DC power input of the power conditioner has been proposed as the monitoring parameter. However, the value from Pmp or DC power output is always significantly smaller than that of classical method using Isc as the monitoring source.

One of the possible reasons is that non-uniform illumination onto the cell result from off-axis concentration. Since fill factor $F F$ depends on uniformity of illumination and $P m p$ is also affected of $F F$, the drop of $F F$ by misalignment further reduces Pmp. However, it was shown that it is not an only cause of the deterioration of acceptance angle in $\mathrm{Pm}$. The experiment was done by modules with DFK optics (Zamora Herranz et al., 2012; Datas, 2013; Nagai et al., 2014). DFK optics is excellent optics of $1000 \mathrm{X}$ concentration (lens to cell ratio with assemble margin) and $1234 \mathrm{X}$ concentration (lens to cell ratio of designed aperture area) with keeping uniformity under plus or minus 1 degree of acceptance angle range (Zamora Herranz et al., 2012; Datas, 2013; Nagai et al., 2014). In spite of its uniformity in illumination onto solar cells, the module acceptance angle by Pm is smaller than that of Isc (Herrero et al., 2010). Precise measurement of optics (individual unit) was done in UPM (Universidad Politécnica de Madrid) and it was shown that it was valid that the illumination was uniform in spite of large acceptance angle range (Nagai et al., 2014). It was also shown that the acceptance angle of the single optics/cell pair did not differ from between Isc and Pmp.

A hint was found in the analysis by MOA (Module Optical Analyzer) (Herrero et al., 2013; Herrero et al., 2010). It looked like that the deterioration of module acceptance angle came from the difference of the positions of the pointing vectors among optics/cell pairs in the module (Herrero et al., 2010). It was also shown that the deterioration of module acceptance angle also came from difference level of assemble control (Hall et al., 2011). These two facts implied that the variation of misalignment levels of optics/cell

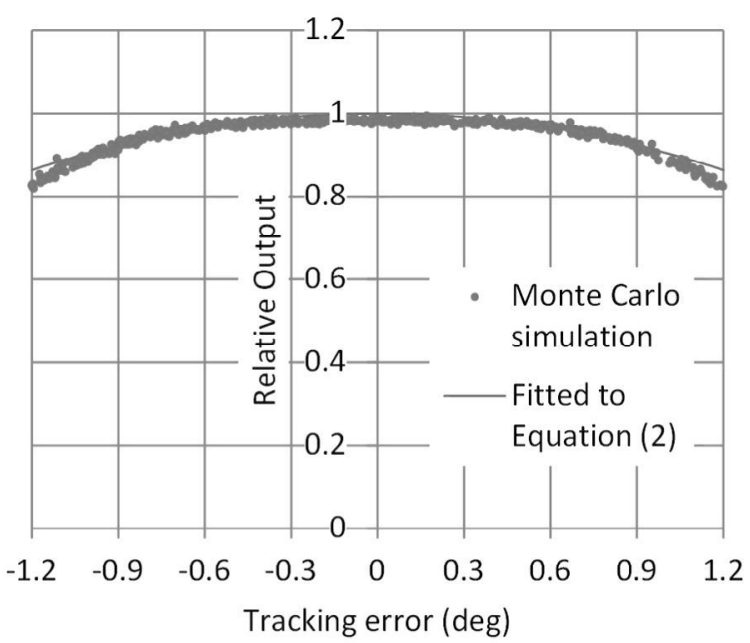

Fig. 5. Fitted result to the Eq. (2) from predicted output from the Monte Carlo simulation (Araki et al., 2016a). 
pairs affects behavior of module to off-tracking and it was thought possible to treat by Monte Carlo simulation in general way.

At first it is important to note that influence to acceptance angle of the module, namely $\delta / \Delta$ after normalization of tracking error, and regardless it is on ISC or P $m p$, is a function of level of assemble error, number of cells that is connected in series in the module, and this characterization parameter $m$.

In this analysis, the level of assembly error was represented by normalized standard deviation of misalignment in optics/cell pair $\sigma / \Delta$ where $\sigma$ is standard deviation of misalignment angle in the sample group consists of the same number of the cells that is connected in series in the module, and $\Delta$ is acceptance half angle of the optics (defined at $90 \%$ output). In the 1-D model, it was shown that $\sigma / \Delta$ value had a strong correlation to the degradation of module performance by bad process control (Araki et al., 2016a). The

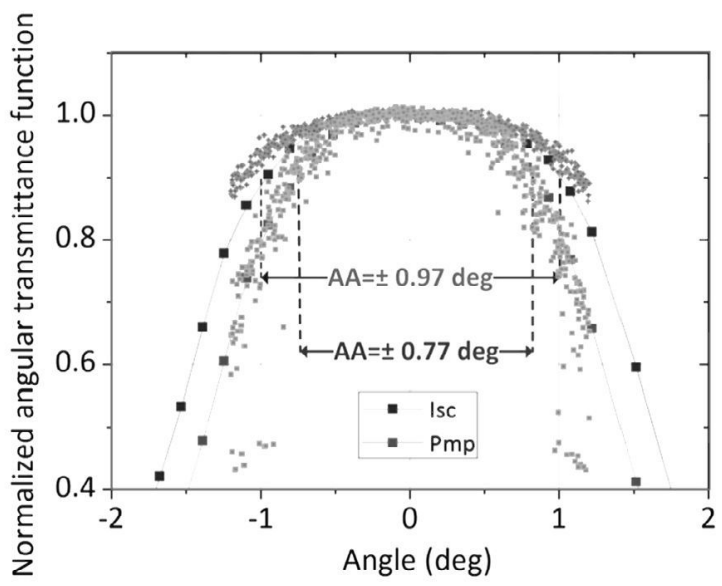

Fig. 6. Comparison of the measured and simulated acceptance angles of the CPV module. Bigger square symbols are measured data points and smaller dots are simulated data points. characterization parameter of optics $m(m=3.445, \Delta=1$ degree in the direction parallel to cell sides, and $m=3.077, \Delta=1.05$ degree in the direction parallel to the diagonal of the cell) and number of cells in a single string (series connected) inside the module $N$ $(N=36)$ were set to that of DFK module.

One of the calculated examples of the synthesized off-axis characteristics curve of the CPV module is plotted in Fig. 5. Eq. (3) was used for fitting $\mathrm{m}$ and $\Delta$ to Eq. (2). The above calculation was compared to the measured data. The comparison result is shown in Fig. 6. Both trend of ISc and Pmp measured acceptance were very closed to the simulated ones.

The calculated results of the synthesized angular transmittance of CPV module as a function of normalized misalignment $\sigma / \Delta$ is shown in Fig. 7.

As it is shown in Fig. 4, when it was assumed assemble was done without any assemble errors, difference of module acceptance angle in between Isc and Pmp was not significant, even with including component variance. However, with the increase of variance of assemble errors, acceptance angle of Pmp gradually decreased while that of Isc increased. The increase of acceptance angle of $I s c$ with the increase of misalignment errors was measured by other CPV system (Antón and Sala, 2004).

This trend is summarized in Fig. 8. This chart may be useful to anticipate the real acceptance angle of the module with given production control. After measurement of 20 modules, namely 1440 measurements of misalignment of the lens/cell pair in both $X$ and $Y$ direction, and direct measurement of $\Delta$ of a single set of the optics and cell, the $\sigma / \Delta$ of the prototype line was measured to be as $\sigma / \Delta=0.233$ in arbitrary directions. The anticipated acceptance angle of the module by Pmp was 0.76 degree, while the measured value was 0.77 degree.

The trend of ISC was also compared (Fig. 9). At the point of $\sigma /$ $\Delta=0.233$, the anticipated angle of acceptance angle by Isc was 1.04 degree, while the measured one was 0.97 degree.

It can be said that the prediction by the new Monte Carlo simulation met the measured acceptance angle of the module in both ISC and Pmp.

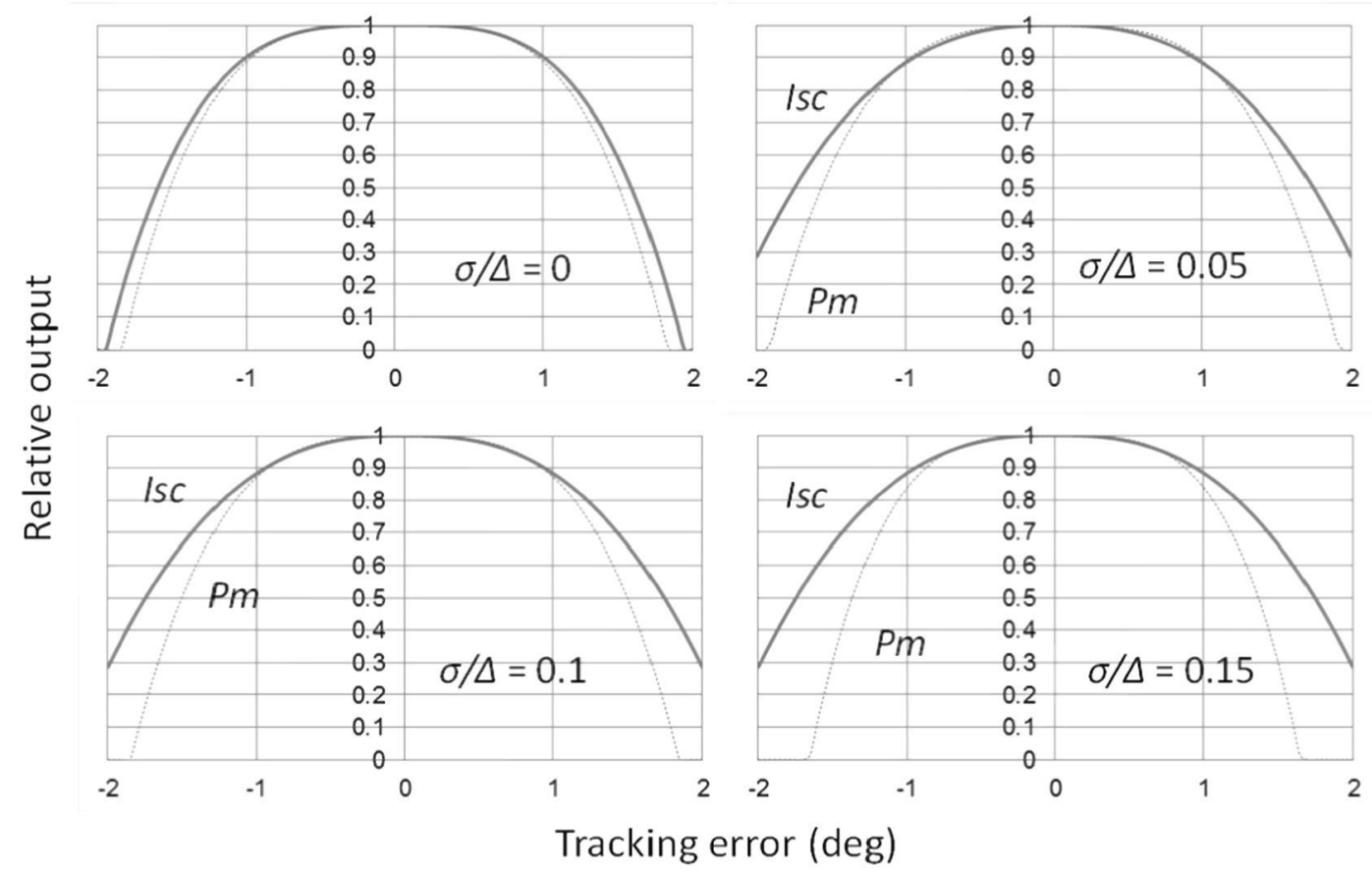

Fig. 7. Simulated result of the synthesized centroid by Pmp and Isc with different levels of $\sigma / \Delta$ (Araki et al., 2016a). 


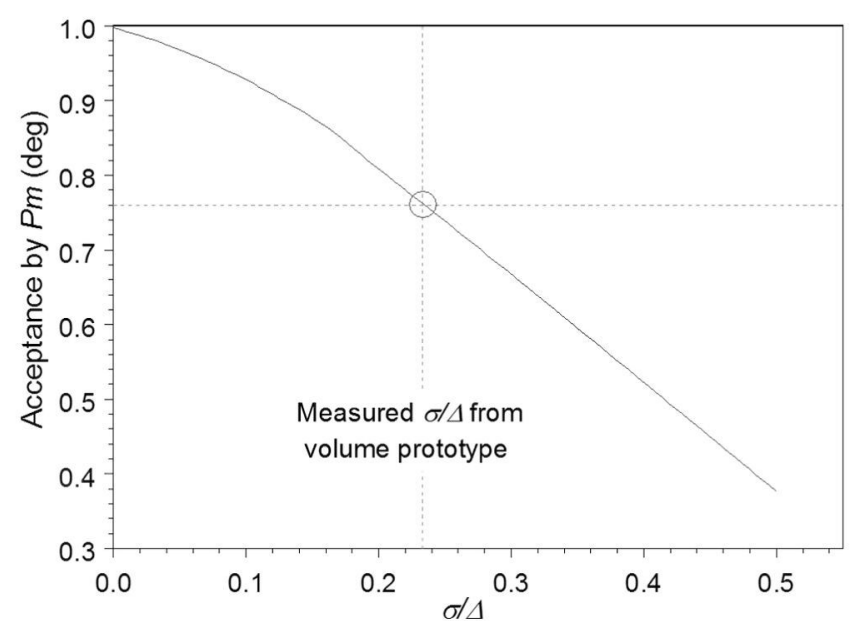

Fig. 8. Anticipation of deterioration of the module acceptance angle of Pmp (defined at $90 \%$ of the power point) at as a function of the level of the parameter assemble error $\sigma / \Delta$.

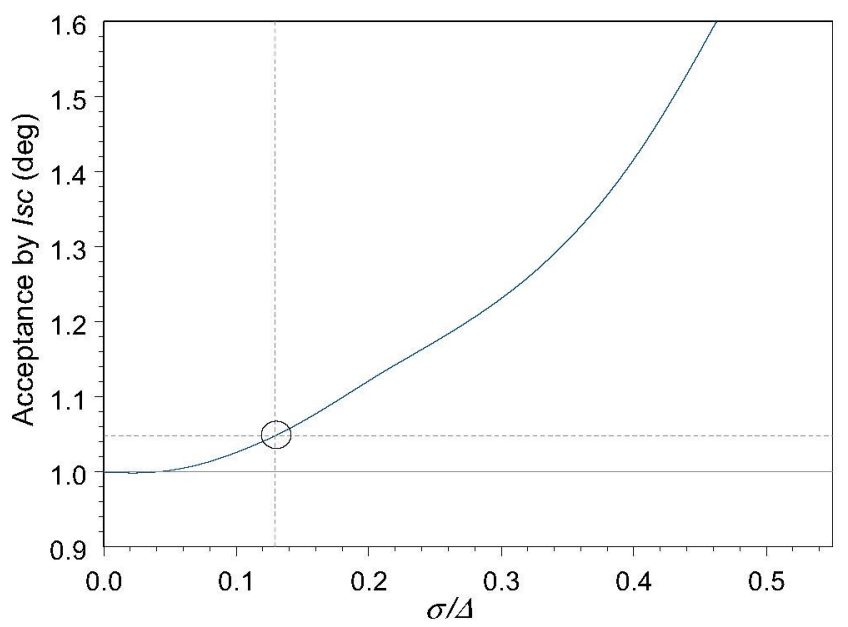

Fig. 9. Anticipation of deterioration of the module acceptance angle of Isc (defined at $90 \%$ of the current) at as a function of the level of the parameter assemble error $\sigma /$ $\Delta$.

\subsection{Loss of the acceptance angle by poor process control}

Next, the Monte Carlo simulation was applied to the two groups of modules with the different levels of misalignment control in assembly. The result is shown in Table 1 . The line $\mathrm{A}$ assembled modules carefully by an experienced technician and the line B assembled modules only by the instruction and the drawings. $\sigma /$ $\Delta$ is the normalized standard deviation of assemble errors relative to the designed acceptance half angle of the $90 \%$ power point of the lens/cell pair. It was shown that the calculated acceptance angles in both levels of tolerance control were much closed to those of measured values.

Table 1

Measured and calculated acceptance angles in different production lines.

\begin{tabular}{llll}
\hline Line & $\sigma / \Delta$ & \multicolumn{2}{l}{ Acceptance angle (degree) } \\
\cline { 3 - 4 } & & Calculated & Measured $(\mathrm{N}=1)$ \\
\hline A & 0.12 & \pm 0.942 & \pm 0.92 \\
B & 0.33 & \pm 0.722 & \pm 0.71 \\
\hline
\end{tabular}

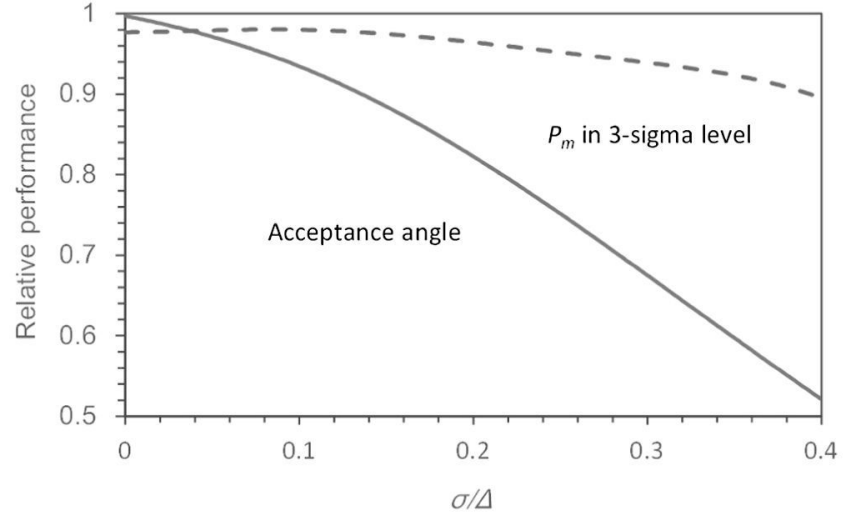

Fig. 10. Impact of the assembly error to the power loss of the module and the acceptance angle. The power loss is scaled by the relative output (Pmp) of the module in the worst performance in the 3-sigma level. The acceptance angle is defined as the $90 \%$ of the power point. The X-axis is the ratio of the standard deviation of assembly errors $\sigma$ divided by the designed acceptance half angle $\Delta$ ( $90 \%$ power point). The distribution of assembly errors was assumed as a normal distribution with an average of 0 degree of assembly error. The number of the cells connected in series is 36 and the characterization parameter of the optics $m$ is 3.445 in 0 degree and 3.077 in 45 degree.

\subsection{Comparison to $1-D$ result}

Assembly error is responsible to both the power loss of the module and the loss of the acceptance angle. The former loss is anticipated by either 1-D or 2-D Monte Carlo simulation (Araki et al., 2016a) but the latter is needed to be calculated by the 2-D Monte Carlo simulation (Araki et al., 2016b).

Fig. 10 compares impact of the assembly error to the power loss of the module and the acceptance angle. The power loss was evaluated by the worst performance in the 3-sigma level (Araki et al., 2016a). Except for very precise assembly error control $(\sigma /$ $\Delta<0.03$ ), the impact of the assembly error was mainly observed in the loss of the acceptance angle.

The rough rule for acceptable assembles misalignment is that the standard deviation of assemble misalignment level will be less than $10 \%$ of acceptance angle of the optics, whereas that of power output is $20 \%$.

Unfortunately, the acceptance angle of the CPV modules is seldom inspected in the module production line while that of the power loss is commonly inspected by the solar simulators. This fact implies that the performance ratio of the CPV system may often drop by the invisible tracking error when the combination of the module and the tracker was combined with insufficient design margin. It is highly encouraged to add the measurement of the acceptance angle to the inspection items (Herrero et al., 2014).

\section{Conclusion}

Assembly error is responsible to both the power loss of the module and the loss of the acceptance angle. The former loss can be anticipated by the 1-D Monte Carlo simulation, but the 2-D simulation is necessary for analysis of the acceptance angle.

The 2-D Monte Carlo simulation successfully and quantifiably explained the reason why the acceptance angle of the CPV module measured by Pmp is smaller than that of Isc. It is also validated by the measurement of the acceptance angle in two different production line with different levels of assembly-error control.

The impact of the assembly error was mainly observed in the loss of the acceptance angle, rather than the power loss. 


\section{Acknowledgments}

This work has been partially supported by NEDO in Japan and by the European Commission through the funding of the project NGCPV EUROPE JAPAN PROJECT (EU Ref. No 283798). 